\title{
Topological and fractal aspects of the main hydraulic parameters
}

\author{
Anton Lyapin ${ }^{1 *}$ and Valery Lyapin ${ }^{2}$ \\ ${ }^{1}$ Moscow state university of civil engineering, Yaroslavskoye shosse, 26, Moscow, Russia, 129337 \\ ${ }^{2}$ Journal «Power Technology and Engineering», 2/1 Voroncovsky Pereulok, Moscow, Russia
}

\begin{abstract}
The traditional concept of wetted cross-section, wetted perimeter and hydraulic radius seems fairly simple and clear, especially in the case of smooth boundaries of the flow. However, for the rough boundary surface covered with randomly arranged roughness the definition of the wetted cross-section as a normal to all elementary streams becomes notably problematic. More controversial seems the concept of wetted perimeter. This issue is of particular importance for the beds with high-dimensional roughness for which the geometric dimensions of the flow and the roughness elements are of the same order. The article shows that topological features of the boundary surfaces for the natural channels allow definitely refer them to fractals. Further study of the bed roughness based on its fractal nature can form a new approach to methodological soundness of main hydraulic parameters and open up new opportunities for their description.
\end{abstract}

Key words: hydraulics, hydraulic resistance, wetted cross-section, wetted perimeter, hydraulic radius.

\section{Introduction}

Hydraulic calculations of pipe and open channel flow in the practice of hydraulic engineering involve the following tasks:

- Definition of the size of the watercourse based on the specified discharge;

- Determining the capacity of the watercourse in terms of its size.

In engineering calculations based on one-dimensional flow model are used averaged flow characteristics: the average over the cross section of flow velocity $\mathrm{V}$ and the depth $\mathrm{h}$. The calculation task is to determine the Chezy coefficient (C), or uniquely associated hydraulic resistance coefficient $\lambda=8 \mathrm{~g} / \mathrm{C}^{2}$ ( $\mathrm{g}-$ acceleration due to gravity). The relationship of these factors with the flow and channel elements called the hydraulic resistance law. To determine the coefficients $\mathrm{C}$ and $\lambda$ there are a large number of empirical and semi-empirical dependences comprising the hydraulic radius $\mathrm{R}$ as the characteristic linear dimension of the watercourse. The exponential formula (Manning, Pavlovsky and

\footnotetext{
* Corresponding author: lyapinanton@gmail.com
} 
others) with constant or variable exponent "y" are the most common type for the open channels [1-2]:

$$
\mathrm{C}=\frac{1}{n} R^{y} .
$$

Obviously, the hydraulic radius $\mathrm{R}$ used in the hydraulic resistance laws should have a clear and understandable methodological substantiation. The classic definition is the following: "hydraulic radius is the ratio of the wetted cross-sectional area $\Omega$ to the wetted perimeter $\chi$ of the bed" $[1,2,3]$

$$
R=\frac{\Omega}{\chi} .
$$

The standard explanation of the $R$ calculations often reduced to simple schemes, such as

$$
\begin{aligned}
\chi= & b+2 h ; \\
R & =\frac{b h}{b+2 h} .
\end{aligned}
$$

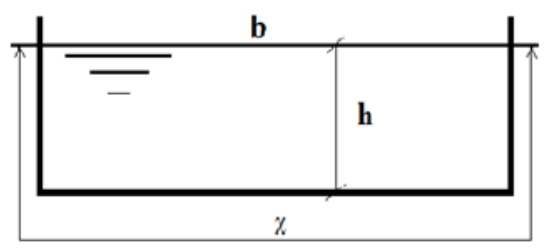

Fig.1. The open channel flow design scheme

Used herein the concepts of channel wetted cross-section, wetted perimeter and hydraulic radius, seem simple and clear especially in the case of "smooth" surface. The flow in this virtual "smooth" bed taken as consisting of elementary streams, which is a necessary justification for the using of the traditional interpretation of the wetted crosssection: wetted cross-section must be normal to all elementary stream tubes (or elementary streams) [2-3]. However, in practice we usually observe the rough surfaces often with a significant roughness for example, hydraulic tunnels traversed by drilling and blasting method. It is impossible to find a smooth contour, even in the case of usual water pipes (Fig. 2) provided with a corresponding increase.

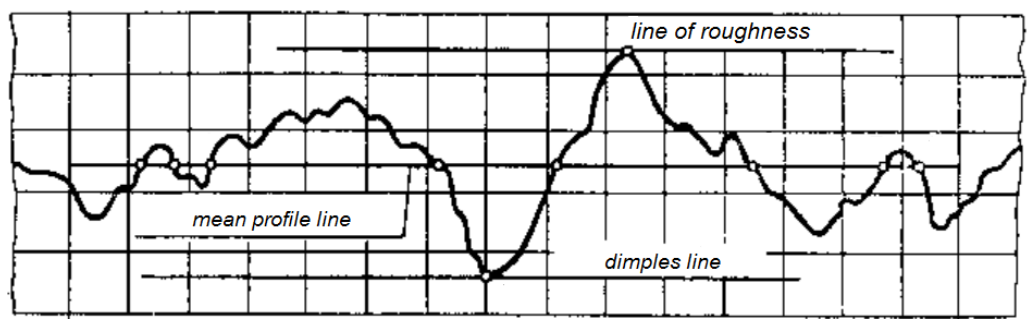

Fig. 2. The fragment of the inner surface profile of the concrete pressure pipe [4]

The eddy zones forming in the near bed region of rough channels [1], are the cause the specific flow around roughness. In these zones (zones of influence of the roughness), the flow cannot be considered as a flow consisting of elementary streams and there is uncertainty associated with the original concept of the wetted cross-section. This issue becomes important for the beds with increased roughness for which the geometric dimensions of the watercourse and the roughness elements are of the same order. Therefore, 
the definition of the wetted cross-section boundaries of the flow, which defined like the normal to all elementary streams, is very problematic.

More difficult is the case with the introduction of the concept of the wetted perimeter. Its current wording - "wetted perimeter is the part of the wetted cross-section perimeter along which the liquid contacts the bed of the channel" [1-3].

\section{Application of fractal theory}

Unfortunately, when the so ingrained concept was realized nothing had been known about fractals. The term "fractal" created by B.B.Mandelbrot from the Latin participle fractus [5]. The corresponding verb frangere means "break, crush, and create fragments of irregular shape". Under a stricter definition of a fractal fall sets for which dimension Hausdorff Besicovitch is strictly greater than its topological dimension.

Consider as an example Fig. 2 and focus on the curve the envelope of the roughness elements. At first glance, this curve seems like each roughness element has a clearly defined contour at each point of which one we can draw a tangent. However, in subsequent approximation clearly revealed an increasing number of shape incorrectness and original clearness and the apparent smoothness of the curve would disappear. Boundary contour curve would include all the smaller irregularly shaped elements, which exclude the possibility of the existence of a tangent to the contour. In the absence of function's derivatives describing a continuous contour of the bed surface will not be surprising because "In nature all curves having no tangent could be considered as a rule, while the right curves - such as for example a circle - interesting but very special case» [5]. The length of the considered boundary curve will tend to an unlimited increase. This is because the curve has a very irregular shape and further approximation increases number of smaller parts, which in turn increases the overall length unlimitedly. Infinite length of the wetted perimeter of the channel confirms its fractal nature and raises questions about the lawfulness of the original concept of the wetted perimeter. The above-mentioned features of the boundary surface contour is also characteristic for natural riverbeds that certainly allows attributing them to the Mandelbrot fractal sets.

Obvious examples of fractals are also the coastlines of islands, mountain ranges forms and so on [5] (Fig. 3).

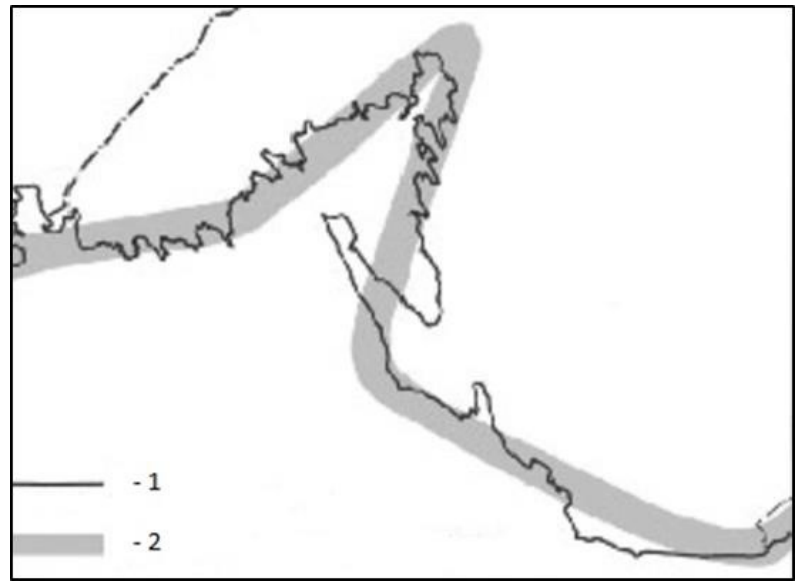

Fig. 3. The fragment of the Vyborg Gulf coastline (Russia) [6]

1- map scale of 1:500 000;2- map scale of 1:22 000000 
Comparing Fig. 2 and 3 indicates the outward similarity of the contours in their respective degrees of approximation. These topologically similar lines including bed boundary contours nevertheless have different fractal dimension that promotes their further identification.

Fractal aspects consideration allows to move on to the next level of roughness description compared with a standard approach of Euclidean Geometry limited height, shape and mutual arrangement of the roughness elements. Considering that the development of a new approach to the evaluation of bed roughness given its fractal nature the subject of future research we accept as a possible alternative the standard idealization in determining the length of the wetted perimeter of the rough bed to use channel with conditionally smooth bed.

I.Nikuradze [7] used this approach for the processing of experimental data received from pipes with sand roughness. He proposed to use as a flow model the flow in a pipe with smooth walls and the effect of roughness to consider in the form of the actual reduction of the wetted cross-section area. The diameter of the conventionally smooth pipe determined volumetrically. Thus, I.Nikuradze retained the ability to use the classical concepts of the wetted cross-section, wetted perimeter and hydraulic radius interpreting them for the virtual equivalent pipe with conditional smooth walls.

The transition to the model of "smooth" bed determined now as a choice of a method for calculating the conditional size (depth) of the flow. The lack of generally accepted methodology for calculating and obtaining experimental data greatly complicates the comparison of the numerous studies results. The most common is the equivalent roughness especially for pipes with natural or irregular roughness. However, the elementary analysis of different interpretations of this approach [7] reveals the absence of a harmonized concept of equivalent roughness. Most often the key conditions for transition from a given pipe to the equivalent pipe Nikuradze with sand roughness are:

- equality of the hydraulic resistance coefficients $(\lambda)$ in quadratic resistance zone;

- equality of the head losses for a given pipe and the equivalent pipe with sand roughness.

Experience shows that the using of these two procedures does not lead to equality obtained through them results. Consequently, this approach needs further clarification and harmonization.

For open rough channels historically formed two basic approaches to the issue of what is meant by the line of contact of the flow with a rough bed and consequently the location of the zero reference plane:

The study of topological (geometric) features of the boundary surfaces to determine the some "average" value of the height of roughness elements (roughness parameter). This approach is still be using in open channel hydraulics when determining roughness coefficient (Surface roughness in the hydraulic calculation of open channels is determined by the special tables linking its value to the characteristic features of the channel, including, as major factors linear dimensions and shape of the roughness).

Search a virtual flow boundary near the rough bed using analytical (graphic) approach naturally specifying its form without taking into account the roughness contour (for example a circle, rectangle, trapezoid and the like). This approach uses a geometric factors (the volumetric method) as well as the combination of geometric and hydrodynamic parameters (determination the location of a zero reference plane using local velocity distribution, etc.) [8]. 


\section{Conclusions}

1. Unfortunately, the problem of choosing the location of zero reference plane is still far from being resolved. In particular, checking calculations performed for artificial roughness in the form of isosceles triangles [10] revealed the conventionality of the standard approach and appreciable divergences in the calculations of the hydraulic radius even in the case of infinitely small roughness elements.

2. This study marked the existence of gaps in the methodological foundations of the pipe and open channel hydraulics associated with the definition of the key design parameters.

3. The proposed approach to the evaluation of bed roughness based on its fractal nature eliminates the inconsistency of the original concepts of the wetted cross-section and wetted perimeter and opens up new possibilities for their description.

4. A complex combination of topological and fractal dimensions, obviously, allows to reach a higher quality of descriptions of roughness in comparison with Euclidean geometry limited by form, height and relative position of the roughness elements

\section{References}

1. V.T. Chow, Open channel hydraulics (Moscow, Stroyizdat, 1969)

2. A.D. Altschul, P.G. Kiselev, Hydraulics and aerodynamics (Moscow, Stroyizdat, 1987)

3. R.R. Chugaev, Hydraulics (Leningrad, Energoizdat, 1982)

4. GOST 12586.0-83. Reinforced concrete pressure pipes. Specifications. (Moscow, 1994)

5. Benoit B. Mandelbrot. Fractal geometry of nature (New York: W. H. Freeman, 1983)

6. K.S. Lazarevich, Geography, 31, 10-12 (2004)

7. J. Nikuradze, Forsch. Arb. Ing.-Wes., Heft 361, 2-22, 1931

8. V.U. Lyapin, Hydrotechnical Construction, 5, 42-44, 1994

9. Liapin V.U. Hydraulic resistance of gradually varied nonuniform and uniform flow in open channels (2004)

10. L.I. Vysotsky and others, Interuniversity scientific collection, Saratov, State Tech. Univ., 49-51 (2011) 